\title{
¿QUIÉN CANTA A MALENA?
}

\section{WHO SINGS TO MALENA?}

\begin{abstract}
Manuel González Ponisio
manuelgonzalezponisio@gmail.com
\end{abstract} Instituto de Investigación en Producción y Enseñanza del Arte Argentino y Latinoamericano. Introducción al Lenguaje Musical. Producción y Análisis III. Facultad de Bellas Artes Universidad Nacional de La Plata. Argentina

Recibido: 10/12/2017 | Aceptado: 9/4/2018

\section{RESUMEN}

El siguiente trabajo aborda tres versiones del tango Maleno tomando como eje principal la práctica improvisada entendida como un modo de producción identitario, frecuente tanto en el tango como en otras músicas populares latinoamericanas.

\section{PALABRAS CLAVE}

Interpretación; versión; práctica; improvisación; popular

\section{ABSTRACT}

This paper deals with three Moleno tango versions taking the improvised practice understood as identity production as the focus of analysis, which is frequent both in tango and in other instances of Latin American popular music.

\section{KEYWORDS}

Interpretation; version; practice; improvisation; popular 
El siguiente trabajo tiene como objetivo abordar tres versiones del tango Molena. Por un lado, proponemos utilizar la categoría de práctica improvisado (González, 2015) entendiendo que abarca aquello que ocurre cuando un músico interpreta una obra previamente compuesta, teniendo en cuenta que en las músicas populares latinoamericanas la composición previa tiene distintos grados de especificidad. Es decir, que no nos referimos a la improvisación en los términos que suele presentarse en el jozz, ya que allí se destaca una búsqueda formal distinta a la que se desarrolla en otros ámbitos. Por otro lado, entendemos que la interpretoción desde su óptica tradicional europea no abarca los cambios en las alturas, los desplazamientos rítmicos, los cambios en las articulaciones que resignifican los materiales de una música. Incluso, veremos que las prácticas que ocurren en las músicas populares latinoamericanas, entre ellas el tango, pondrán en duda la idea de origen de la obra, ya que el constante devenir de las distintas puestas en escena de la obra irán modificándola y resinificándola. Esto hará que nos preguntemos ¿cuál es la melodía original? Sobre todo cuando un músico presente distintas versiones de la misma obra, aun siendo de composición propia, muchas veces con el aporte de distintos músicos en cada versión. Aquí se visualizará la idea de construcción colectiva, donde lo individual se desdibuja y pone en discusión la idea tradicional de composición como algo terminado y definitivo. A su vez, tomaremos aspectos ligados a las condiciones de producción y las construcciones sociales y culturales propias de la zona del Río de la Plata.

\section{IMPROVISACIÓN}

Cada vez que un músico improvisa lo hace en un contexto que le da un marco determinado. En las músicas populares este marco suele construirse a partir de los géneros, de los contextos de producción, de las ideas o las pautas que proponen los mismos músicos antes de comenzar a tocar o mientras lo hacen, de las ideas o las pautas que proponen aquellos que dirigen 0 conducen el entorno en que se está produciendo música. Entendiendo la categoría de género como un campo amplio que constantemente redefine sus límites, podemos decir que algunos de ellos le dan a la improvisación un lugar de mayor importancia, mientras que otros le asignan una carga negativa o bien la ocultan. En algunas músicas populares urbonas la improvisación es un factor que contiene rasgos que le dan identidad al género, que reproducen formas de aproximación a dicha improvisación, al tiempo que permiten innovaciones que lo amplían, modifican o resignifican. Tal es el caso del jazz o el choro, y en algunos casos el rock. Quizás sea este el lugar común para hablar de la improvisación. En algunas instancias, la idea centroeuropea de genio creador se vincula con este tipo de improvisación, especialmente en el jazz, donde los improvisadores son valorados desde 
una perspectiva individual, en ocasiones desvinculados de los aspectos colectivos y separados de los colegas que producen en otros espacios o que los acompañan en la producción; es decir, aquellos compañeros que tocan e interactúan en un mismo grupo. Esta perspectiva ha sido revisada por autores, como Ingrid Monson (1996) o David Lines (2005); sin embargo, en el imaginario colectivo sigue presente la idea del músico-individuo-virtuoso con relación al género mencionado.

Existen otros espacios en los que la producción se da de forma improvisada, ya sea por condiciones coyunturales, por búsquedas estéticas o por formas de producción preestablecidas, por ejemplo, en el folclore argentino, cuando se presentan un guitarrista o un bombista que acompaña a un cantante en una peña folclórica. Tanto quienes acompañan como quien canta una canción elegida en el momento mismo de tocar acuden a recursos adquiridos previamente y los usan de forma más o menos creativa. Parten, entonces, de lo que Wade Matthews (2012) Ilama «modelo discursivo» al hacer alusión a los materiales y a los recursos que se construyen alrededor de un género. Entendemos que se trata de una práctica improvisada. Aquí vemos condiciones coyunturales atravesadas por un espacio de encuentro social que influye en la producción, en vínculo con el público que tiene una participación activa. En el rock no suelen utilizarse registros escritos previos a la producción en el ensayo. La construcción compositiva y de arreglo se da muchas veces de forma colectiva y colaborativa, donde los músicos prueban, proponen, improvisan. Tanto en el rock como en el folclore aquello que se trabaja en el ensayo de forma más o menos improvisada suele convertirse en arreglo y llega un punto en el que ya no se modifica. Es decir que al momento de presentarse en vivo ya no hay grandes espacios para la improvisación en términos de búsquedas novedosas. Sin embargo, esta práctica forma parte del proceso de producción.

Matthews (2012) hace referencia al músico improvisador y destaca que hay al menos tres planos de interrelación: la interacción con el género, la interacción con los demás músicos y la interacción con el público. Estos planos dialogan y se confunden unos con otros a tal punto que no siempre es fácil diferenciarlos. No obstante, aquí el género es visto como algo acabado con el cual se dialoga. ¿Pero no son los mismos músicos los que realizan aportes para la construcción del género? Esta pregunta nos lleva a vincular la categoría de género con la de identidad. La noción de identidad ha sido objeto de grandes discusiones a partir del auge de la globalización y la posmodernidad. Las corrientes posmodernas presentaron críticas al esencialismo que, si bien permitieron repensar aspectos vinculados a las etnias y a la vieja noción de raza, posicionó al mercado como una entidad que desplazó al Estado y promovió un multiculturalismo en donde fue difícil establecer categorías asequibles. Ticio Escobar (2004) toma de Slavok Zizek su crítica al multiculturalismo posmoderno: 
[...] el multiculturalismo se convierte en la forma ideal de la ideología del capitalismo global; entonces, la nueva comunidad universal, pos EstadoNación, construye, a través del mercado global, su propia ficción hegemónica de tolerancia multiculturalista [...] (p. 67).

El mercado funciona como regulador de las producciones musicales en distintos puntos del planeta e impone sus propias reglas. Stuart Hall (2003) presenta el concepto de identidad como portador de múltiples categorías que no se unifican y son construidas de distintas maneras a través de discursos, prácticas y posiciones diferentes, a menudo cruzadas y antagónicas. Estas categorías están sujetas al desarrollo histórico, lo que posibilita un constante proceso de cambio y de transformación. Desde este punto de vista, la noción de identidad aplicada a la categoría de género nos permite tener una mirada más amplia a partir de la cual pensar los procesos y los materiales que forman parte de su construcción. Asimismo, tener en cuenta los procesos históricos nos permite entender aspectos musicales concretos. Por ejemplo, en el tango, las grandes orquestas que surgieron en los años cuarenta y ampliaron la cantidad de violines y bandoneones y sumaron a la viola tuvieron la posibilidad de financiar grupos más numerosos debido al ascenso social experimentado en la Argentina durante el peronismo. De igual manera, podemos observar el surgimiento de los dúos de guitarra y bandoneón en los años sesenta, no como una búsqueda estética, sino, más bien, como un intento de supervivencia de los músicos que ya no podían trabajar con sus orquestas en un contexto económico desfavorable.

\section{TANGO}

Resulta sustancial para el análisis del tango su relación con la tradición. En este sentido, Stuart Hall (2003) asevera que la identidad se relaciona tanto con la invención de la tradición como con la tradición misma. Esta propuesta nos lleva a evitar leerla como una reiteración incesante y observar un constante devenir que presenta lo mismo que cambia, idea que toma de Paul Gilroy (2003). En otras palabras, no es el presunto retorno a las raíces, sino una aceptación de nuestros múltiples caminos. Cuando aparece el quinteto de Diego Schissi o la Orquesta Fernandez Fierro (OFF) la tradición tanguera de los años dorados es interpelada. La posibilidad de ser lo mismo que cambia es puesta en acto.

Se presentan aquí dos problemas. Uno es la concepción del tango actual que el mercado intenta manejar y que lo proyecta como música transcultural, con rasgos latino-europeizados posibles de ser abordados por orquestas sinfónicas de todo el mundo ¿Qué queda allí del tango? ¿En algún momento deja de serlo? Aquí la necesidad del mercado de definir un género de bateas o de nichos de internet entra en conflicto con las nuevas propuestas. 
Por otro lado, se presentan los núcleos históricos, muchas veces vinculados al auge de las grandes orquestas, aunque también al cantante con cuarteto de guitarras gardeleano, que luego fueron tomados como los mitos que se proyectaron sobre la idea de tango. Sin embargo, el nuevo siglo trajo nuevas voces que, al tiempo que dialogaron con la tradición tanguera, se preguntaron por sí mismas, como actores activos de su momento histórico. Tomando los ejemplos antes mencionados nos encontramos con un Diego Schissi que, luego de grabar discos de jazz, se volcó al tango y lo hizo partiendo de la formación tradicional de quinteto - piano, bandoneón, guitarra, violín y contrabajo- y le asignó al timbre un valor determinante. Una orquesta que frente a la ausencia de lugares para tocar en los años finales del siglo pasado, donde el tango tenía pocos lugares en Buenos Aires y un público reducido y principalmente compuesto por turistas, comenzó a tocar en las calles de San Telmo. Actualmente, tiene un auditorio propio, el Club Atlético Fernández Fierro, y toca a sala llena. También resuelve su producción artística y ejecutiva como cooperativa siguiendo el legado de Osvaldo Pugliese. Ambas propuestas, junto con muchas otras, tomaron aspectos de la tradición tanguera y reformularon otros. La OfF partió de la formación de orquesta típica y tomó aspectos rítmicos y tímbricos con constantes repeticiones que parecen emular un loop producido por una loptop, y puso en primer plano una voz rota, casi hablada (postgoyenecheana). Allí, el desarrollo melódico-armónico de tradición romántico-europea y las floridas variaciones del bandoneón brillan por su ausencia. Diego Schissi, en una línea que podríamos llamar vanguardista o rupturista, llama a muchas de sus músicas tongos. Lo hace porque, dice, no llegan a ser tangos.

\section{TRES VERSIONES DE MALENA}

Como dijimos antes, tomaremos tres versiones de Moleno, tres casos que analizaremos comparativamente. El primer caso es el de Rubén Juárez en el programa Encuentro en el Estudio (Canal Encuentro, 2015). Allí, sobre el final del programa, el bandoneonista presenta la posibilidad de improvisar a partir de un tango dado. Pide al conductor del programa que elija un tango al azar y éste elige el tango Molena, de Lucio Demare. El bandoneonista propone el uso de materiales propios del género para desarrollar una improvisación. Comienza tocando la melodía original; "primero toco la melodía como es» (Juárez en Canal Encuentro, 2015), dice textualmente Juárez. Aquí se presentan varias preguntas: ¿cómo es ese tango? ¿Cómo lo escribió su autor en la edición para piano? ¿Cómo la tocó este en sus primeras versiones? ¿Cómo la tocaron los referentes más destacados? ¿Cómo establecemos cuál es la versión más significativa? ¿La más conocida por el público? ¿la más conocida por los músicos? ¿la más difundida por las industrias culturales? 
Si nos referimos a la versión escrita tenemos un problema estructural. Sabemos que estas ediciones eran tomadas de las partituras presentadas a la Sociedad Argentina de Autores y Compositores (SADAIC) y que muchas veces representaban la línea melódica en un formato simplificado en el campo rítmico, a veces sin articulación, y con una versión armónica dada por la mano izquierda que funcionaba como una síntesis similar a la escritura de cifrado. En el caso de Malena, la partitura de 1941 presenta en la mano izquierda un desarrollo importante, marcando síncopas y contramelodías, y en la melodía de la mano derecha presenta cambios de articulación. Sin embargo, en la grabación del mismo Demare ${ }^{1}$ de 1942 con el cantante Juan Carlos Miranda (Cantando Tangos, 2010) podemos observar cómo la interpretación excede aquello que aparece escrito. Éste será nuestro segundo caso. Si tomamos sólo la introducción podemos observar que algunas contramelodías del registro del bajo presentes en la partitura son tocadas y otras no. Incluso, una de ellas aparece un compás después con relación al registro de la partitura. Lo mismo podemos decir de la interpretación de los violines, que ornamentan la melodía y modifican el ritmo. Esto se suma a la contramelodía - también llamada armonía - de los mismos violines, que irrumpe y se posiciona en un primer plano y deja atrás durante varios compases a la melodía principal tocada por los bandoneones, lo cual le da un lugar preponderante que no se ve representado en la partitura. Estas contramelodías por lo general hacen notas largas que contrastan con la melodía principal y son ejecutadas por los violines. Si tenemos en cuenta que muchas veces estas contramelodías aparecían escritas con una tipografía más pequeña, podemos decir que podría haber estado presente, pero no lo está. Especulamos con la idea de que esta contramelodía no existiera al momento de presentar la partitura y sea obra del arreglador de turno, en este caso Máximo Mori. Luego, cuando aparece el cantante, la partitura pasa a ser una referencia lejana. Aquí las cuerdas suman una gran cantidad de contramelodías, variaciones melódicas, y a veces duplican a la voz del cantante. Los bandoneones quedan relegados a enriquecer el marcato del piano. El cantante interpreta la melodía con inflexiones en las alturas y pequeñas ornamentaciones. El ritmo melódico está siempre desplazado y, de esta manera, retrasa, levemente la rítmica de la melodía. Muchos aspectos musicales sólo serían posibles de ser llevados a una partitura con técnicas no tradicionales.

La versión de ese mismo año que grabó Aníbal Troilo con su orquesta junto al cantante Francisco Fiorentino (Troilo \& Orquesta Típica con Francisco Fiorentino, 2013) será nuestro tercer caso. Sabemos por distintos relatos de músicos que tocaron con él que Aníbal Troilo le daba muchas indicaciones

1 Demare, quien tocaba el piano, tenía en su orquesta al bandoneonsita Máximo Mori quien se encargaba de hacer los arreglos musicales. La fila de bandoneones se completaba con otros tres integrantes, cinco violines y un contrabajo.

6 Clang 
a los cantantes, por lo cual es probable que la interpretación por parte de Fiorentino sea una síntesis de sus ideas con los acuerdos con el director. La orquesta estaba formada por cinco bandoneones, con Astor Piazzolla como primer bandoneonista solista, cuatro violines, el piano a cargo de Orlando Goñi y el contrabajo. Si bien la función del piano siempre fue importante y estuvo ligada a la dirección de la orquesta de tango, Troilo siempre brindó un espacio para que el pianista hiciera una parte de la melodía que le permitiera desarrollar aspectos interpretativos propios. En esta versión, Orlando Goñi presenta la primera sección de la parte A del tango Moleno en el piano. Allí realiza sutiles desplazamientos rítmicos, que le da un sentido distinto a la melodía con relación a lo que ocuriirá luego con los violines. Además, suma una voz paralela en sextas en la segunda semifrase y luego hace un cambio de registro tocando el final de su sección en el registro grave. Es notable cómo realiza cambios sutiles en las intensidades. Luego, toman la melodía los violines para dejar finalmente a los bandoneones que hacen la melodía con una articulación en marcato. En esta última parte se agrega como material la contramelodía o armonía en los violines. En este caso recae en la última semifrase en lugar de la anteúltima, como ocurre en la versión de Demare. Llegando al final de la canción aparece una pequeña sección en que Piazzolla toca el comienzo de la parte A. Allí se destacan las aproximaciones cromáticas y algunos desplazamientos rítmicos más osados, si lo comparamos con el caso de Goñi. Sobre el final realiza una variación que se transforma en contramelodía cuando toma el rol principal la voz de Francisco Fiorentino.

Podemos observar que, en general, las diferencias presentes en la partitura entre una melodía más lírica, explicitada con un ligado de expresión y una melodía más rítmica, explicitada con acento en cada nota, son respetadas tanto por la orquesta de Triolo como la del propio Demare, pero esto ocurre sólo cuando una fila completa de la orquesta toca la melodía. ES decir que la referencia de la partitura es tenida en cuenta, pero sólo cuándo varios músicos tocan juntos.

La versión de la orquesta de Troilo tuvo mucho éxito y, junto con la aparición de la versión de Demare en la película El viejo Hucho (1942), dirigida por su hermano Lucas Demare para la cual fue compuesta, dio inicio a un derrotero que incluye innumerables versiones. La letra de Homero Manzi tuvo mucha repercusión, por lo cual la mayoría de las versiones tienen voz cantada.

Volveremos ahora al primer caso, la versión de Rubén Juárez. Como dijimos anteriormente, primero establece que va a tocar el tango como es. Aquí, Juárez no parece tener pretensiones de establecer cuál es la forma correcta de tocar Molena y sí de dar un contexto formal a partir del cual realizar su improvisación. Sin embargo, allí el intérprete toca la melodía y la armonía con una serie de particularidades que resultan asignables a su visión de la melodía, su versión del tango Moleno. Allí, Juárez presenta la 
melodía con desplazamientos rítmicos. Estos desplazamientos son más extremos que en las versiones vistas hasta aquí y se destacan al relacionarse con un acompañamiento en negras (marcato) en la mano izquierda del bandoneón, que nunca se detiene, lo cual permite escuchar con claridad los desplazamientos que parecen imitar a un cantante (más cercano al último Goyeneche que a Fiorentino o Miranda), al tiempo que establecen una doble temporalidad: por un lado, el acompañamiento estático y, por otro, una melodía errática. Al mismo tiempo, el plano estático del acompañamiento remite a la función bailable del tango, que en este contexto queda como una referencia lejana. También se destacan los ornamentos con cromatismos, octavas sucesivas y grupos rítmicos tales como tresillo y quintillos.

Inmediatamente después de tocar la primera versión de Malena toca la versión improvisada. Comienza con un desarrollo melódico que sólo puede asociarse a la melodía original por sus direccionalidades. A partir de allí presenta recursos constitutivos del tango. En general hay un desarrollo de gran dominio técnico del instrumento. Podríamos asociar su propuesta a las variaciones de bandoneón, que proponen un espacio en el cual la densidad cronométrica se pone a disposición de la destreza técnica de la fila de bandoneones, aunque estos no sean improvisados. Es llamativo que aquí use la nota blue, característica en el blues o en el jazz, que asocia, así, la improvisación a estos géneros.

En los términos que aquí estamos presentando, la primera sería una próctica improvisado y la segunda, una improvisación. La primera presenta ornamentaciones, arrastres, aproximaciones cromáticas, desplazamientos rítmicos, uso de octavas melódicas (recurso comúnmente usado en el bandoneón por los músicos de tango) y hasta modifica la parte final de la melodía con relación al original. Todos estos elementos se realzan si lo comparamos por la impresión de la partitura original para piano de Lucio Demare, pero también con las versiones del propio Lucio Demare y de Aníbal Troilo. La sección presentada como la parte improvisada utiliza recursos más complejos y, a nuestros fines, menos interesantes. Entraría dentro de los parámetros de la improvisación ya que no hay una referencia explícita a la melodía del tango, sino un desarrollo de materiales que surgen del género pero no de este tango en particular.

\section{CONCLUSIONES}

La práctica improvisada en sus distintas versiones aparece continuamente en la práctica profesional del músico popular. Muchas veces se mezcla con partes escritas o con ideas transmitidas de oído. Creemos que al darle visibilidad podemos observar aspectos que forman parte del proceso creativo en la producción de las músicas populares latinoamericanas. Atravesado por las condiciones en que se presentan en distintos ámbitos,

8 Clang 
podemos ver cómo se materializa de formas disímiles e incluso nos deja frente a la posibilidad de decir que difícilmente todas estas prácticas puedan conformar un todo uniforme. Sin embargo, en cada contexto en que se presenta nos abre la posibilidad de pensar en nuevas dimensiones de las músicas que abordamos. En definitiva, se trata presentar una herramienta que nos ayude a pensar nuestras músicas y tendrá sentido en la medida que no se solidifique ni tenga pretensiones absolutistas.

Podemos establecer que esta práctica no fue tenida en cuenta por los análisis tradicionales. Creemos que esto ocurrió por traer consigo tradiciones que han transitado caminos lejanos a los que se fueron canonizando en la academia. En la actualidad existe un gran interés por la improvisación en los estudios musicales, especialmente en los poscognitivistas. Sin embargo, son escasas las referencias a las condiciones de producción y a la relación con las tradiciones que atraviesan el hecho musical. Entendemos que es necesario hacer el esfuerzo de establecer estos cruces, sea en relación a lo que llamamos prócticas improvisadas o a otras categorías que resulten más útiles a los fines planteados.

Además, el análisis de las prócticas improvisadas nos presenta la posibilidad de repensar los procesos de enseñanza-aprendizaje de estas músicas. Las prácticas vistas llevadas al campo académico, que en nuestra región actualmente toma a las músicas populares latinoamericanas como parte de su currícula, nos posibilitan un acercamiento desde una de sus formas de producción concreta. También nos pone frente a la dificultad de evaluarlo, lo cual requerirá nuevas herramientas para su abordaje.

Desde esta perspectiva, los casos analizados nos presentan varios campos que se entremezclan. En primera instancia, la relación con su origen y la construcción de tradiciones y perspectivas históricas en el tango, atravesado por constantes disputas. Esto se vincula a la construcción colectiva, que pone en crisis el paradigma del compositor-individuo. La mirada tecnicista presenta a una persona que solo se dedica a componer y aquello que compone no tiene lugar a nuevos aportes formales. El compositor es nombrado y no puede perderse entre intérpretes dispersos en el tiempo, en la historia. Es por esta razón que la construcción formal del colectivo pasa a tener una entidad que necesitamos observar para entender cómo funciona la creación artística en este contexto. Al mismo tiempo, la ausencia de la escritura en gran parte del repertorio de músicas populares latinoamericanas le da a la obra un carácter más dinámico que alimenta las nuevas interpretaciones.

En el caso del tango se dio una escritura poco detallada. Si bien podemos encontrar casos como el de Salgán o Piazzolla, donde la escritura adquirió un desarrollo particular, en general podemos decir que la interpretación en el tango requiere un conocimiento del género sin el cual la escritura tiene un corto alcance. Puntualmente el caso de Molena nos da la posibilidad de observar una obra con un largo desarrollo histórico y observar una versión 
que ocurre casi setenta años después de las primeras versiones. Esto nos da una perspectiva en la cual observamos cambios, relecturas y cercanías. Finalmente, diremos que las formas de producción en el tango vistas desde una perspectiva actual presentan la posibilidad de tomar decisiones estéticas y posicionamientos propios como parte constitutiva del devenir histórico, aquel que mantiene materiales, tradiciones, al mismo tiempo que las transforma y resignifica.

\section{REFERENCIAS}

Canal Encuentro. (7 de enero de 2015). Rubén Juórez. Molena Improvisado HD [Archivo de video]. Disponible en https://www.youtube.com/watch?$\mathrm{V}=8 \mathrm{RfHEOQI89E}$

Demare, L. (Director) y Manzi, H., Petit de Murat, U. (Guionistas). (1942). El viejo Hucho [Película]. Argentina: Artistas Argentinos Asociados.

Escobar, T. (2004). El arte fuero de sí. Asunción, Paraguay: FONDEC y CAV, Museo del Barro.

González, M. (2015). La Práctica Improvisatoria en la Música Popular Latinoamericana. Arte e Investigoción, (11), 59-66. Recuperado de http:// papelcosido.fba.unlp.edu.ar/ojs/index.php/aei/article/view/28

Hall, S. y du Gay, P. (2003). Cuestiones de identidad cultural. Avellaneda, Argentina: Amorrortu.

Lines, D. (2005). Music Education for the New Milenium [La Educación Musical para el Nuevo Milenio]. Oxford, Inglaterra: Blackwell Publishing.

Mathews, W. (2012). Improvisando. La libre creación musical. Madrid, España: Turner Publicaciones S.L.

Monson, I. (1996). Saying something: jazz improvisation and interaction. Chicago, Estados Unidos: The University of Chicago Press

Troilo, A. y Orquesta Típica con Francisco Fiorentino. (10 de agosto de 2013). Aníbal Troilo y Orquesto Típica con Francisco Fiorentino. Malena [Archivo de video]. Disponible en https://www.youtube.com/watch?v=m83c-t0089w Cantando Tangos. (20 de noviembre de 2010). Lucio Demare. Juan Carlos Miranda. Malena. Tango (1. versión de 1942). [Archivo de video]. Disponible en https://www.youtube.com/watch?v=VbWcgUbg/y8 